\title{
Existence of Solutions to a Class of Navier Boundary Value Problem Involving the Polyharmonic
}

\author{
Yongyi Lan \\ School of Sciences, Jimei University, Xiamen, China \\ Email: 200661000135@jmu.edu.cn
}

How to cite this paper: Lan, Y.Y. (2018) Existence of Solutions to a Class of Navier Boundary Value Problem Involving the Polyharmonic. Advances in Pure Mathematics, 8, 373-379.

https://doi.org/10.4236/apm.2018.84020

Received: January 23, 2018

Accepted: April 14, 2018

Published: April 17, 2018

Copyright $\odot 2018$ by author and Scientific Research Publishing Inc. This work is licensed under the Creative Commons Attribution International License (CC BY 4.0).

http://creativecommons.org/licenses/by/4.0/

\section{Abstract \\ This paper is devoted to the following high order elliptic problems under the Navier boundary condition: $\left\{\begin{array}{ll}-(\Delta)^{p} u=\lambda f(x, u), & x \in \Omega, \\ u=\Delta u=\cdots=(\Delta)^{p-1} u=0, & x \in \partial \Omega .\end{array}\right.$ Without} assuming the standard subcritical polynomial growth condition ensuring the compactness of a bounded (P.S.) sequence, we show that the Navier boundary value problem has at least a weak nontrivial solution for all $\lambda>0$ by using mountain pass theorem.

\section{Keywords}

Navier Boundary Value Problem, Polyharmonic, Variational Methods, Mountain Pass Theorem

\section{Introduction and Main Results}

Consider the Navier boundary value problem involving the polyharmonic:

$$
\begin{cases}-(\Delta)^{p} u=\lambda f(x, u), & x \in \Omega, \\ u=\Delta u=\cdots=(\Delta)^{p-1} u=0, & x \in \partial \Omega .\end{cases}
$$

where $p \in \mathbb{Z}^{+}=\{1,2,3, \cdots\}, \Omega$ is an open bounded domain in $\mathbb{R}^{N}(N \geq 2 p+1)$ with smooth boundary $\partial \Omega, f(x, t)$ is continuous on $\bar{\Omega} \times \mathbb{R}$. We look for the weak solutions of (1.1) which are the same as the critical points of the functional $I_{\lambda}: H_{\theta}^{p}(\Omega) \rightarrow \mathbb{R}$ defined by

$$
I_{\lambda}(u)=\frac{1}{2}\|u\|^{2}-\lambda \int_{\Omega} F(x, u) \mathrm{d} x,
$$


where $F(x, t)=\int_{0}^{t} f(x, s) \mathrm{d} s$, and

$$
H_{\theta}^{p}(\Omega):=\left\{u \in H^{p}(\Omega): \Delta^{i} u=0 \text { if } x \in \partial \Omega \text {, for } i<\frac{p}{2}\right\}
$$

is denoted by $E_{p}$ and its dual spaces by $E_{p}^{\prime}$. The Hilbert space $E_{p}$ is equipped with the standard scalar product and norm, respectively, given by:

$$
\langle u, v\rangle= \begin{cases}\int_{\Omega}\left(\Delta^{l} u\right)\left(\Delta^{l} v\right) \mathrm{d} x, & \text { if } p=2 l, \\ \int_{\Omega}\left(\nabla \Delta^{l} u\right)\left(\nabla \Delta^{l} v\right) \mathrm{d} x, & \text { if } p=2 l+1,\end{cases}
$$

and

$$
\|u\|=\langle u, u\rangle^{\frac{1}{2}} .
$$

$I_{\lambda}$ is of $C^{1}$ with derivatives given by

$$
\left\langle I_{\lambda}^{\prime}(u), v\right\rangle=\langle u, v\rangle-\lambda \int_{\Omega} f(x, u) v \mathrm{~d} x, u, v \in E_{p} .
$$

Motivated by the fact that such kinds of problems (1.1) are often used to describe an important class of physical problems, many authors have widely developed various methods and techniques, such as variational method, critical point theory, lower and upper solutions, and Brower degree for looking for multiple solutions of elliptic equations involving biharmonic, $p$-biharmonic and polyharmonic type operators (see, for example [1]-[8] and the references therein). In [1], the author considered the existence of positive solutions in semilinear critical problems for polyharmonic operators and proved the existence result in some general domain under the appropriate assumptions by topological methods. In [2], by variational methods, they obtain the existence of multiple weak solutions for a class of elliptic Navier boundary problems involving the p-biharmonic operator. By using Nehari manifold, Y.Y. Shang and L. Wang [3] considered (1.1) with critical growth and sublinear perturbation and obtained the existence of multiple nontrivial solutions. Moreover in [3], a concrete example of application of such mathematical model to describe a physical phenomenon is also pointed out. By three critical points theorem obtained by B. Ricceri, Li and Tang [4] have obtained the existence of at least three weak solutions for a class of Navier boundary value problem involving the p-biharmonic. In [5], combining the mountain pass theorem together with fountain theorem and with local linking theorem and symmetric mountain pass theorem, $\mathrm{Li}$ and Tang establish the existence of at least one solution and infinitely many solutions for a class of $p(x)$-biharmonic equations with Navier boundary condition, respectively. By using critical point theory, the authors [6] establish the existence of infinitely many weak solutions for a class of elliptic Navier boundary value problems depending on two parameters and involving the p-biharmonic operator. In [7], a Navier boundary value problem is treated where the left-hand side of the equation involves an operator that is more general than the $p$-biharmonic. Further, by using the abstract and technical approach developed in [8] [9] [10] the authors are interested in looking for the existence of infinitely many weak solutions 
of perturbed $p$-biharmonic equations. For more results about (1.1) and its variants, we refer the interested readers to [11]-[16] and the references therein.

The aim of the manuscript is to consider the problem in a different case: based on the mountain pass theorem; we show that the Navier boundary value problem has at least a weak nontrivial solution for all $\lambda>0$. We now assume the nonlinearity $f(x, t)$ satisfies the following conditions.

$\left(\mathrm{F}_{1}\right)$ There exist constants $\alpha \geq 0, \beta \geq 0$ such that

$$
t f(x, t)-2 F(x, t) \geq \alpha|f(x, t)|^{\frac{2 N}{N+2 p}} \text { for all } x \in \bar{\Omega}, \forall|t|>\beta .
$$

$\left(\mathrm{F}_{2}\right)$

$$
\lim _{|t| \rightarrow \infty} \frac{f(x, t)}{|t|^{p^{*}-1}}=0 \text { uniformly a.e. } x \in \Omega .
$$

where $p^{*}=\frac{2 N}{N-2 p}$ is the Sobolev critical exponent.

$$
\lim _{t \rightarrow 0} \frac{f(x, t)}{t^{2 p-1}}=0 \text { uniformly a.e. } x \in \Omega \text {. }
$$

$\left(\mathrm{F}_{4}\right)$

$$
\lim _{|t| \rightarrow+\infty} \frac{F(x, t)}{t^{2 p}}=+\infty \text { uniformly a.e. } x \in \Omega .
$$

The main theorem of this paper reads as follows.

Theorem 1. Suppose that $\left(\mathrm{F}_{1}\right)-\left(\mathrm{F}_{4}\right)$ hold. Then Equation (1.1) has a weak nontrivial solution, for all $\lambda>0$.

This paper is organized as follows. In Section 2, we give the proof of the Theorem 1. In the sequel, the letter $C$ or $C_{i}(i=0,1,2,3, \cdots)$ will be used to denote various positive constants whose exact value is irrelevant.

\section{Proof of Theorem 1}

Proof of Theorem 1. We will divide the proof of the theorem in three steps.

First step: the (P.S.) condition. We only prove Theorem 1 by taking $\lambda=1$. The case of a general $\lambda>0$ will follow immediately. In fact, if $\lambda>0$ and $\lambda \neq 1$, we only let $g(x, t)=\lambda f(x, t)$. Then (1.1) becomes

$$
\begin{cases}-(\Delta)^{p} u=g(x, u), & x \in \Omega, \\ u=\Delta u=\cdots=(\Delta)^{p-1} u=0, & x \in \partial \Omega,\end{cases}
$$

The nonlinear term $g$ also satisfies condition $\left(\mathrm{F}_{1}\right)-\left(\mathrm{F}_{4}\right)$, then the same conclusion as in the case $\lambda=1$ holds. We denote $I_{1}$ as $I$ for convenience.

Let $\left\{u_{n}\right\}$ be any sequence in $E_{p}$ such that $I\left(u_{n}\right)$ is bounded and $\left\|I^{\prime}\left(u_{n}\right)\right\|$ converges to zero, that is,

$$
I\left(u_{n}\right) \rightarrow C,\left\|I^{\prime}\left(u_{n}\right)\right\| \rightarrow 0 .
$$

Applying the Riesz-Fréchet representation theorem, there is $v_{n} \in E_{p}$ such 
that

$$
\left\langle I^{\prime}\left(u_{n}\right), \phi\right\rangle=\left\langle v_{n}, \phi\right\rangle, \forall \phi \in E_{p} \text {, and }\left\|I^{\prime}\left(u_{n}\right)\right\|=\left\|v_{n}\right\| .
$$

Thus, we obtain

$$
\left\langle u_{n}-v_{n}, \phi\right\rangle=\int_{\Omega} f\left(x, u_{n}\right) \phi \mathrm{d} x, \forall \phi \in E_{p} .
$$

In (2.1), taking $\phi=u_{n}-v_{n}$, we obtain

$$
\left\|u_{n}-v_{n}\right\|^{2}=\int_{\Omega} f\left(x, u_{n}\right)\left(u_{n}-v_{n}\right) \mathrm{d} x .
$$

From Hölder's inequality, we get:

$$
\left\|u_{n}-v_{n}\right\| \frac{2 N}{N+2 p} \leq C_{1} \int_{\Omega} \mid f\left(x, u_{n}\right)^{\frac{2 N}{N+2 p}} \mathrm{~d} x .
$$

On the other hand, since

$$
\int_{\Omega}\left(u_{n} f\left(x, u_{n}\right)-2 F\left(x, u_{n}\right)\right) \mathrm{d} x=2 I\left(u_{n}\right)-\left\langle I^{\prime}\left(u_{n}\right), u_{n}\right\rangle .
$$

then $\left(\mathrm{F}_{1}\right)$ together with $2 I\left(u_{n}\right)-\left\langle I^{\prime}\left(u_{n}\right), u_{n}\right\rangle \leq C_{2}\left(\left\|u_{n}\right\|+1\right)$, we have

$$
\begin{aligned}
& \int_{\Omega} \mid f\left(x, u_{n}\right)^{\frac{2 N}{N+2 p}} \mathrm{~d} x \\
& \leq C_{3} \int_{\Omega}\left(u_{n} f\left(x, u_{n}\right)-2 F\left(x, u_{n}\right)\right) \mathrm{d} x \\
& =2 I\left(u_{n}\right)-\left\langle I^{\prime}\left(u_{n}\right), u_{n}\right\rangle \\
& \leq C_{4}\left(\left\|u_{n}\right\|+1\right) \\
& \leq C_{5}\left(\left\|u_{n}\right\|+1\right)^{\frac{2 N}{N+2 p}} .
\end{aligned}
$$

Since $\left\|I^{\prime}\left(u_{n}\right)\right\|=\left\|v_{n}\right\|$ is bounded and $\frac{2 N}{N+2 p}>1$, by (2.2) and (2.4), we conclude that $\left\|u_{n}\right\|$ is bounded.

By $\left(\mathrm{F}_{1}\right)$, for any $\varepsilon>0$ there exists $a(\varepsilon)>0$ such that

$$
|f(x, t)|^{\frac{2 N}{N+2 p}} \leq\left.\varepsilon|t|\right|^{\frac{2 N}{N-2 p}}+a(\varepsilon) \text { for } t \in \mathbb{R}, x \in \Omega .
$$

Since $\left\{u_{n}\right\}$ is bounded in $E_{p}$ and $f(x, t)$ is continuous on $\bar{\Omega} \times \mathbb{R}$, then there exists a subsequence of $\left\{u_{n}\right\}$, still denoted by $\left\{u_{n}\right\}$ and $u \in E_{p}$ such that

$$
\int_{\Omega} f\left(x, u_{n}\right) \mathrm{d} x \rightarrow \int_{\Omega} f(x, u) \mathrm{d} x .
$$

Moreover there is an $M>0$ such that

$$
|f(x, t)| \leq M \text {, for all }(x, t) \in \bar{\Omega} \times \mathbb{R} .
$$

Combining (2.6) and (2.7) with the Egorov theorem, there exists $\Omega_{\varepsilon} \subseteq \Omega$ such that $\left|\Omega \backslash \Omega_{\varepsilon}\right|<\frac{\varepsilon}{a(\varepsilon)}$ and $\left|f\left(x, u_{n}\right)-f(x, u)\right|_{L^{\infty}\left(\Omega_{\varepsilon}\right)}$ converge to 0 , and further

$$
\int_{\Omega_{\varepsilon}}\left|f\left(x, u_{n}\right)-f(x, u)\right|^{\frac{2 N}{N+2 p}} \mathrm{~d} x \rightarrow 0 .
$$


Also, since $\left\{u_{n}\right\}$ is bounded in $E_{p}$, we derive that

$$
\int_{\Omega}\left(\left|u_{n}\right| \frac{2 N}{N-2 p}+|u|^{\frac{2 N}{N-2 p}}\right) \mathrm{d} x \leq C_{4} .
$$

On one hand, we have

$$
\begin{aligned}
& \int_{\Omega \Omega_{\varepsilon}}\left|f\left(x, u_{n}\right)-f(x, u)\right|^{\frac{2 N}{N+2 p}} \mathrm{~d} x \\
& \leq 2^{\frac{2 N}{N+2 p}}\left(\int_{\Omega \mid \Omega_{\varepsilon}}\left|f\left(x, u_{n}\right)\right|^{\frac{2 N}{N+2 p}}+|f(x, u)|^{\frac{2 N}{N+2 p}} \mathrm{~d} x\right) .
\end{aligned}
$$

Combining (2.5) and (2.9) with the fact $\left|\Omega \backslash \Omega_{\varepsilon}\right|<\frac{\varepsilon}{a(\varepsilon)}$, one has

$$
\int_{\Omega \backslash \Omega_{\varepsilon}}\left|f\left(x, u_{n}\right)-f(x, u)\right|^{\frac{2 N}{N+2 p}} \mathrm{~d} x \leq C_{5} \varepsilon .
$$

By (2.8), there exists an integer $N(\varepsilon)$ such that for $n>N(\varepsilon)$ we have

$$
\int_{\Omega_{\varepsilon}}\left|f\left(x, u_{n}\right)-f(x, u)\right|^{\frac{2 N}{N+2 p}} \mathrm{~d} x \leq \varepsilon .
$$

Taking account of (2.10), for $n>N(\varepsilon)$, we have

$$
\int_{\Omega}\left|f\left(x, u_{n}\right)-f(x, u)\right|^{\frac{2 N}{N+2 p}} \mathrm{~d} x \leq\left(C_{5}+1\right) \varepsilon .
$$

This proves that $u_{n} \rightarrow u$ in $E_{p}$.

Second step: mountain-pass geometric structure.

$I$ has a mountain pass geometry; i.e., there exists $u_{1} \in E_{p}$ and constants $r, \rho>0$ such that $I\left(u_{1}\right)<0,\left\|u_{1}\right\|_{p}>r$ and

$$
I(u) \geq \rho \text {, when }\|u\|_{p}=r \text {. }
$$

Indeed, using the assumption $\left(\mathrm{F}_{3}\right)$, we have $t_{0}>0$ and $\mu_{0} \in\left(0, \mu_{1}\right)$ such that

$$
\frac{f(x, t)}{t^{2 p-1}}<\mu_{0} \text {, for }|t|<t_{0}
$$

where $\mu_{1}$ denotes the lowest eigenvalue of $(-\Delta)^{p}$ with the Navier boundary condition. As shown in [16], $\mu_{1}>0$ is the principal eigenvalue of

$$
\begin{cases}(-\Delta)^{p} u=\mu u, & x \in \Omega, \\ u=\Delta u=\cdots=(\Delta)^{p-1} u=0, & x \in \partial \Omega,\end{cases}
$$

and there is a corresponding eigenfunction $\phi_{1}>0$ in $\Omega$.

Hence, by (2.14), we have

$$
F(x, t) \leq \frac{\mu_{0}}{2} t^{2 p} \text {, for }|t| \leq t_{0} .
$$

This inequality together with the assumption $\left(\mathrm{F}_{2}\right)$ implies that

$$
F(x, t) \leq \frac{\mu_{0}}{2} t^{2 p}+C|t|^{p^{*}}, \text { for } t \in \mathrm{R}
$$

with some $C>0$. Since $\mu_{1}>0$ denotes the lowest eigenvalue of $(-\Delta)^{p}$ with the Navier boundary condition, it follows that $\|u\|_{p}^{2} \geq \mu_{1}\|u\|_{p}^{2}$ for $u \in E_{p}$. Then 
the following estimates hold:

$$
\begin{aligned}
I(u) & \geq \frac{1}{2}\|u\|_{p}^{2}-\frac{\mu_{0}}{2}\|u\|_{p}^{2}-C\left\|u_{n}\right\|_{p^{*}}^{p^{*}} \\
& \geq \frac{\mu_{1}-\mu_{0}}{2 \mu_{1}}\|u\|_{p}^{2}-C^{\prime}\left\|u_{n}\right\|_{p}^{p^{*}} .
\end{aligned}
$$

This implies that there exist constants $r$ and $\rho$ such that

$$
I(u) \geq \rho \text {, when }\|u\|_{p}=r \text {. }
$$

From $\left(\mathrm{F}_{4}\right)$ follows that, for all $M>0$ there exists $C_{M}>0$, such that

$$
F(x, t) \geq M t^{2 p}-C_{M}, \forall x \in \Omega, \forall t>0 .
$$

By (3.4), we have

$$
\begin{aligned}
I\left(t \phi_{1}\right) & =\frac{1}{2} t^{2}\left\|\phi_{1}\right\|_{p}^{2}-\int_{\Omega} F\left(x, t \phi_{1}\right) \mathrm{d} x \\
& \leq \frac{1}{2} t^{2}\left\|\phi_{1}\right\|_{p}^{2}-t^{2 p} \int_{\Omega} M \phi_{1}^{2 p} \mathrm{~d} x+c|\Omega| \rightarrow-\infty \text { as } t \rightarrow \infty,
\end{aligned}
$$

where $\phi_{1}$ is the principal eigenfunction of (2.15) corresponding to the principal eigenvalue $\mu_{1}$. We fix $t>0$ so large that $I\left(t \phi_{1}\right)<0$ and $t\left\|\phi_{1}\right\|_{p}>r$. Let $u_{1}:=t \phi_{1} \in E_{p}$ and then constants $r, \rho>0$ such that $I\left(u_{1}\right)<0,\left\|u_{1}\right\|_{p}>r$ and satisfies (2.13), i.e. I has a mountain pass geometry.

Last step: critical value of $I$.

For $u_{1}$ in second step, we define

$$
\begin{gathered}
\Gamma:=\left\{\gamma: C[0,1] \rightarrow E_{p} \mid \gamma(0)=0, \gamma(1)=u_{1}\right\}, \\
c_{0}:=\inf _{\gamma \in \Gamma} \max _{0 \leq t \leq 1} I(\gamma(t)) .
\end{gathered}
$$

From Proposition 1, I satisfies the (P.S.) condition. By the Mountain Pass Theorem implies that $c_{0}$ is a critical value of $I$.

\section{Acknowledgements}

This work is supported by Natural Science Foundation of China (No. 11671331); Natural Science Foundation of Fujian Province (No. 2015J01585) and Scientific Research Foundation of Jimei University.

\section{References}

[1] Ge, Y.X. (2005) Positive Solutions in Semilinear Critical Problems for Polyharmonic Operators. Journal de Mathématiques Pures et Appliquées, 84, 199-245. https://doi.org/10.1016/j.matpur.2004.10.002

[2] Molica Biscia, G. and Repovš, D. (2012) Multiple Solutions of p-Biharmonic Equations with Navier Boundary Conditions. Complex Variables and Elliptic Equations, 59, 271-284. https://doi.org/10.1080/17476933.2012.734301

[3] Shang, Y.Y. and Wang, L. (2014) Multiple Nontrivial Solutions for a Class of Semilinear Polyharmonic Equations. Acta Mathematica Scientia, 34B, 1495-1509. https://doi.org/10.1016/S0252-9602(14)60099-0 
[4] Li, C. and Tang, C.L. (2010) Three Solutions for a Navier Boundary Value Problem Involving the $p$-Biharmonic. Nonlinear Analysis, 72, 1339-1347. https://doi.org/10.1016/j.na.2009.08.011

[5] Li, L. and Tang, C.L. (2013) Existence and Multiplicity of Solutions for a Class of $p(x)$-Biharmonic Equations. Acta Mathematica Scientia, 33B, 155-170. https://doi.org/10.1016/S0252-9602(12)60202-1

[6] Candito, P., Li, L. and Livrea, R. (2012) Infinitely Many Solutions for a Perturbed Nonlinear Navier Boundary Value Problem Involving the $p$-Biharmonic. Nonlinear Analysis, 75, 6360-6369. https://doi.org/10.1016/j.na.2012.07.015

[7] Wang, W. and Zhao, P. (2008) Nonuniformly Nonlinear Elliptic Equations of p-Biharmonic Type. Journal of Mathematical Analysis and Applications, 348, 730-738. https://doi.org/10.1016/j.jmaa.2008.07.068

[8] Candito, P. and Molica Bisci, G. (2012) Multiple Solutions for a Navier Boundary Value Problem Involving the $p$-Biharmonic. Discrete and Continuous Dynamical Systems-Series $S$, 5, 741-751. https://doi.org/10.3934/dcdss.2012.5.741

[9] Bonanno, G. and Molica Bisci, G. (2010) A Remark on a Perturbed Neumann Problem. Studia Universitatis Babeș-Bolyai Mathematica, 4, 17-25.

[10] Bonanno, G. and Molica Bisci, G. (2010) Infinitely Many Solutions for a Dirichlet Problem Involving the p-Laplacian. Proceedings of the Royal Society of Edinburgh Section A, 140, 737-752. https://doi.org/10.1017/S0308210509000845

[11] Hangelbroek, T. and Lauve, A. (2014) The Polyharmonic Dirichlet Problem and Path Counting. Journal de Mathématiques Pures et Appliquées, 102, 449-381. https://doi.org/10.1016/j.matpur.2013.12.001

[12] Lazer, A.C. and McKenna, P.J. (1990) Large-Amplitude Periodic Oscillations in Suspension Bridges: Some New Connections with Nonlinear Analysis. SIAM Review, 32, 537-578. https://doi.org/10.1137/1032120

[13] Wang, Y. and Shen, Y. (2009) Infinitely Many Sign-Changing Solutions for a Class of Biharmonic Equations without Symmetry. Nonlinear Analysis, 71, 967-977. https://doi.org/10.1016/j.na.2008.11.052

[14] Yang, Z., Geng, D. and Yan, H. (2006) Existence of Multiple Solutions for a Semilinear Biharmonic Equations with Critical Exponent. Acta Mathematica Scientia, 27, 129-142.

[15] Gazzola, F., Christoph Grunau, H. and Sweers, G. (2010) Polyharmonic Boundary Value Problems. Lecture Notes in Mathematics, Vol. 1991, Springer, Berlin Heidelberg. https://doi.org/10.1007/978-3-642-12245-3

[16] Drabek, P. and Otani, M. (2001) Global Bifurcation Result for the p-Biharmonic Operator. Electronic Journal of Differential Equations, 2001, 1-19. 